\title{
Factors Associated with Unmet Dental Needs among Single-Person Households in Korea
}

\author{
Dong-Hwi Kim and Hyeongmi $\mathrm{Kim}^{\dagger}$ \\ Department of Dental Hygiene, Namseoul University, Cheonan 31020, Korea
}

\begin{abstract}
Background: The purpose of this study is to provide the data for discussions related to oral health promotion policies for single-person households by analyzing the status of unmet dental needs and related factors in single-person households in Korea, based on the Anderson model. Methods: The data, obtained from 544 single-person households of those over 20 years old who were targeted for the 6th Korea National Health and Nutrition Examination Survey, were analyzed through a complex sample frequency analysis, complex sample cross analysis (Rao-Scott chi-square test), and complex sample binary logistic regression analysis on a complex sampling design.

Results: The most frequently given reason for an unmet dental need among single-person households was economic (52.4\%). Factors related to the unmet dental needs of single-person households are smoking, which is a predisposing factor; personal income levels, which are an enabling factor; chewing discomfort; and limited daily activities, which are need factors. Smokers, the high-income group, the chewing-discomfort group, and the limited activity group showed high unmet dental care experience. Smokers had a 2.75 times higher rate of unmet dental care than non-smokers, and the high-income group had a 5.29 times higher rate of unmet dental needs than the median group. The rate of unmet dental needs for the chewing discomfort group was 3.27 times higher than the non-chewing discomfort group, and the limited activity group had a 7.87 times higher rate of unmet dental needs than the non-limited activity group.

Conclusion: It is necessary to map out policies designed to help maintain and promote met dental needs considered to be internally heterogeneous to single-person householders, based on the Anderson model.
\end{abstract}

Key Words: Dental care, Health surveys, Single person, Smoking

\section{Introduction}

Oral health is a factor that can improve overall health and quality of life. Oral diseases are irreversible diseases and diseases with a lower morbidity level tend to have a more favorable prognosis and are treatable at lower cost. However, people often do not receive the necessary dental services at the right time owing to various factors, including economic level, accessibility, and psychological factors, which are referred to as unmet dental needs ${ }^{1)}$. A broader concept is that unmet medical needs include "that which a person wants or a physician has diagnosed as required treatment" and "diseases or disabilities that could have been prevented, mitigated, or eliminated if the medical needs were properly met" needs" is also a concept that stems from these definitions. The level of unmet medical needs is one of the indicators for the accessibility ${ }^{2)}$ and equity of medical services, which is because these needs measure the experience of not being able to utilize the care needed, rather than the absolute quantity of care provided. Increased unmet medical needs can lead to higher mortality rates among the elderly population ${ }^{3)}$, greater disease severity among patients $^{4)}$, and poorer prognosis due to greater likelihood of complications ${ }^{5}$.

The number of dental hospitals continue to increase ${ }^{6}$, while some studies have reported that there is a surplus of dentists $^{7)}$. However, in the 2016 Korean National Health 
and Nutrition Examination Survey (KNHANES) conducted by the Korea Centers for Disease Control and Prevention $(\mathrm{KCDC})^{8}, 22.2 \%$ of the respondents answered "Yes" to the question, "Did you have the experience of not receiving dental treatment you needed in the past year?" This is a higher percentage than the unmet medical needs of $7.7 \%$ reported in the same study ${ }^{8}$. Dental care services include many more treatments that are not covered by the national health insurance, as compared to medical services, which means that dental care services have less health insurance coverage than medical services. Consequently, an individual's ability to pay becomes an important factor in meeting dental care needs, more so than when using medical services. Therefore, it is necessary to pay attention to experiences of unmet dental needs in identifying the factors associated with inequity in dental care utilization due to low coverage.

In Korean society, the highest percentage of household types is a single-person household ${ }^{9}$. This trend appears not only in Korea, but worldwide, and it is forecast to accelerate even more because of changes in social organization and the progression of population aging ${ }^{10}$. The health status of a single-person household member tends to be lower than that of members of other types of households, and thus, "single-person household" itself falls under the category of socially vulnerable groups ${ }^{11)}$. Single-person household members have a higher risk of cardiovascular disease than members of other types of households $^{12)}$ and have a greater propensity for behavior harmful to health such as smoking and drinking ${ }^{13)}$; they also tend to have poorer mental health, including depression ${ }^{14)}$. However, owing to various factors, such as household income and enrollment in regional health insurance ${ }^{15)}$, the medical needs of single-person household tend to go unmet at a higher rate than of members of other types of households ${ }^{16,17)}$.

Oral health represents the first step in the digestive system and is closely associated with overall health. It is also significant that most oral diseases are irreversible and that the experience of unmet dental needs can be a factor that exacerbates poor oral health. However, there is a lack of previous studies on oral health and unmet dental needs among single-person households. Accordingly, the present study aimed to examine the current status of unmet dental needs among single-person households and to analyze the influencing factors. The findings in the present study provide basic data for establishing reasonable public health policies on oral health care among single-person households or identifying measures for reducing unmet dental need experiences among single-person households.

\section{Materials and Methods}

\section{Subjects}

The present study analyzed raw data from the 6th KNHANES (2013 2015) conducted by KCDC. Among a total of 21,724 people in the raw data from KNHANES based on the 6th complex sampling design, there were 1,704 adults aged 20 years or older who were members of a single-person household, of whom 544 people had experienced unmet dental needs. In the study, 1,704 single-person household members were established as the sub-population and analyzed.

\section{Selection of variables}

The dependent variable was set as unmet dental needs experience, namely, an affirmative response to "Did you have experience of not receiving dental treatment you needed in the past year?" in the oral health questionnaire survey. The present study used the rate of unmet dental needs experience, rather than the number of people with unmet dental needs experience.

Independent variables were selected based on the Anderson model, which is widely used in studies on the utilization of dental services ${ }^{18}$. This is a model of personal medical utilization based on three types of factors: predisposing factors that include the characteristics of individuals, enabling factors that enable utilization of medical resources or exert disabilities, and need factors that indicate the severity of disease ${ }^{19}$. The Anderson model is a typical socio-behavioral model used as an analytic framework for predicting predisposing, enabling, and need factors that result in the utilization of medical services ${ }^{20)}$. It is useful when undertaking analysis based on a systematic theoretical framework concerning factors that affect utilization of dental care services among other 
personal factors.

Various factors appearing in KNHANES were classified as predisposing, enabling, and need factors. Two researchers independently classified the variables, and only those that both researchers agreed upon were included. Personal characteristics, such as sex, age, education level, hypertension status, and smoking status, were selected as predisposing factors. Income level (income quartile), type of health insurance, enrollment in private insurance, oral health screening in the past year, toothbrushing the previous day, and economic activity status were selected as enabling factors Subjective oral health status, toothache in the past year, speech impairment, chewing discomfort, periodontal disease, permanent teeth dental caries experience, and activity limitations were selected as need factors that indicate the severity of disease.

The selected variables were calculated to fit a range suitable for the purpose of analyses. Each variable was checked using the user's manual for the 6th KNHANES raw data and missing values were treated as "not known," "excluded," "unable to record," or "no response." Age was divided into groups of $20 \sim 29,30 \sim 39,40 \sim 49,50 \sim 59$, $60 \sim 74$ (early elderly), and $\geq 75$ (late elderly) years. Income level was divided into upper, middle (uppermiddle and lower-middle), and lower groups. Health insurance was divided into regional subscriber, workplace subscriber, and medical aid recipient (class 1 and 2). Smoking status was divided into "yes" (smoker and smoke sometimes) and "no" (former smoker, not applicable, and unknown). Subjective oral health status was divided into good (very good and good), average, and poor (poor and very poor). Details of the variables are shown in Table 1 .

\section{Data analysis}

The present study constructed a research protocol using variance estimation strata and survey households according to the complex sampling design of the 6th KNHANES raw data and, during the analyses, complex sample analyses were used. The weight was calculated by using the weight applied to the health questionnaire and screening. As the raw data were extracted by complex stratified sampling, weights, stratification variables, and cluster variables were applied in the analysis.
Complex sample frequency analysis was performed to investigate the frequency and percentage of single-person households, while Rao-Scott chi-square $\left(\chi^{2}\right)$ test was used to analyze the differences in unmet dental needs experiences among single-person households by predisposing, enabling, and need factors. Moreover, complex sample binary logistic regression analysis was used to analyze the factors associated with unmet dental needs experiences among single-person households, with the number of permanent teeth with dental caries experience adjusted as a covariate. SPSS Statistics (ver. 20.0; IBM Corp., Armonk, NY, USA) was used for statistical analyses with the significance level established at $5 \%$.

Table 1. Definition of Variables

\begin{tabular}{|c|c|}
\hline Variable & Content \\
\hline Unmet dental needs & Yes/no \\
\hline \multicolumn{2}{|l|}{ Predisposing factors } \\
\hline Sex & Male/female \\
\hline Age group (y) & $\begin{array}{l}20 \sim 29 / 30 \sim 39 / 40 \sim 49 / 50 \sim 59 \\
\quad / 60 \sim 74 / \geq 75\end{array}$ \\
\hline Educational level & $\begin{array}{l}\leq \text { Elementary school/middle } \\
\text { school/ high school/ } \\
\geq \text { university }\end{array}$ \\
\hline Hypertension & Yes/no \\
\hline Diabetes mellitus & Yes/no \\
\hline Smoking & Yes/no \\
\hline \multicolumn{2}{|l|}{ Enabling factors } \\
\hline Personal income level & High/middle/low \\
\hline Type of medical insurance & $\begin{array}{l}\text { Community based/employed/ } \\
\text { medical aid }\end{array}$ \\
\hline Private health insurance & Yes/no \\
\hline Residence & Dong/eup, myeon \\
\hline Regular oral check-ups & Yes/no \\
\hline Toothbrushing per day & Yes/no \\
\hline Economic activity & Yes/no \\
\hline \multicolumn{2}{|l|}{ Need factors } \\
\hline Perceived oral health & Good/normal/poor \\
\hline Toothache within a year & Yes/no \\
\hline Speaking difficulty & $\begin{array}{l}\text { Comfortable/unawareness/ } \\
\text { uncomfortable }\end{array}$ \\
\hline Chewing difficulty & Yes/no \\
\hline Periodontal disease & $\begin{array}{l}\text { Yes (periodontal pocket depth } \\
\geq 4 \mathrm{~mm} \text { )/no (periodontal } \\
\text { pocket depth }<4 \mathrm{~mm} \text { ) }\end{array}$ \\
\hline Untreated dental caries & Yes $\left(\mathrm{DT}^{\mathrm{a}} \geq 1\right) /$ no $\left(\mathrm{DT}^{\mathrm{a}}=0\right)$ \\
\hline Limitation of activity & Yes/no \\
\hline
\end{tabular}

${ }^{\mathrm{a}}$ Mean number of decayed permanent teeth (DT). 


\section{Results}

\section{Rate of unmet dental needs experiences among} single-person households

During the survey period (2013 2015), the highest percentage of single-person households was found in 2015 (35.1\%), while the highest rate of unmet dental needs experience among single-person households was found in 2014 (34.3\%) (Table 2). The percentage of single-person households appeared in increasing order from 2013 (31.3\%), to 2014 (33.6\%), to 2015 (35.1\%), while the rate of unmet dental needs experience among single-person households appeared in the order of 2014 (34.3\%), 2013 (32.4\%), and 2015 (33.3\%).

\section{Reasons for unmet dental needs experiences as} perceived by members of single-person households

Among the responses given by member of single-person households as the reason for unmet dental needs experiences, economic reasons were highest at $52.4 \%$ (Table 3). Other reasons appeared in the following order: "felt it was less important than other issues" (17.3\%), "could not miss work or school" (12.7\%), "fear of dental treatment" $(9.5 \%)$, and "too far to travel" (3.3\%).

\section{Reasons for unmet dental needs experiences by age}

With respect to the reasons for unmet dental needs experiences by the age of the member of a single-person household, differences were found between age groups (Table 4). Among people aged 20 29 years, the most common response was "felt it was less important than other issues" (31.4\%). Among people aged 30 39 years, "could not miss work or school" (36.6\%) was the most common response. Among people aged 40 49, 50 59, $60 \sim 74$, and $\geq 75$ years, an "economic reason" was the most common response $(42.9 \%, 58.2 \%, 66.3 \%$, and $61.2 \%$, respectively).

\section{Factors associated with unmet dental needs} experiences among single-person households

Among the predisposing, enabling, and need factors, differences in unmet dental needs experiences among single-person households were found based on smoking status, income level, regular oral health screening, subjective oral health status, toothache in the past year, speech impairment, chewing discomfort, permanent teeth dental caries experience, and activity limitation (Table 5).

Among the predisposing factors, only smoking status showed differences in unmet dental needs experiences. The percentage of smokers and non-smokers who

Table 3. Reason for Unmet Dental Needs of the Single-Person Householder

\begin{tabular}{lrrr}
\hline \multicolumn{1}{c}{ Variable } & \multicolumn{1}{c}{$\mathrm{n}$} & Weighted & \multicolumn{1}{c}{$\%^{\mathrm{a}}(\mathrm{SE})$} \\
\hline Financial burden & 308 & 553,049 & $52.4(2.8)$ \\
Too far to go & 25 & 35,267 & $3.3(0.8)$ \\
Busy with school or work & 46 & 134,416 & $12.7(2.0)$ \\
Poor mobility & 20 & 26,990 & $2.6(0.6)$ \\
No one help with their children & 1 & 2,263 & $0.2(0.2)$ \\
Lower on the priority scale & 80 & 182,886 & $17.3(2.4)$ \\
Fear of dental treatment & 53 & 100,549 & $9.5(1.4)$ \\
Other reasons & 11 & 20,294 & $1.9(0.7)$ \\
Total & 544 & $1,055,715$ & $100(0.0)$ \\
\hline
\end{tabular}

SE: standard error.

${ }^{\mathrm{a}}$ Percentage of weighted population.

Table 2. Status of Unmet Dental Needs of the Single-Person Householder

\begin{tabular}{crrrrrrrr}
\hline \multirow{2}{*}{ Year } & \multicolumn{3}{c}{ Single-person householder } & & \multicolumn{3}{c}{ Unmet dental needs of the single-person householder } \\
\cline { 2 - 4 } & \multicolumn{1}{c}{$\mathrm{n}$} & Weighted & $\%^{\mathrm{a}}(\mathrm{SE})$ & & $\mathrm{n}$ & Weighted & $\%^{\mathrm{a}}(\mathrm{SE})$ \\
\hline 2013 & 531 & $1,003,292$ & $31.3(3.0)$ & & 176 & 342,537 & $32.4(3.7)$ \\
2014 & 573 & $1,074,154$ & $33.6(3.1)$ & & 182 & 361,712 & $34.3(3.6)$ \\
2015 & 600 & $1,124,048$ & $35.1(3.1)$ & & 186 & 351,466 & $33.3(3.6)$ \\
Total & 1,704 & $3,201,494$ & $100(0.0)$ & & 544 & $1,055,715$ & $100(0.0)$ \\
\hline
\end{tabular}

SE: standard error.

${ }^{\mathrm{a}}$ Percentage of weighted population. 


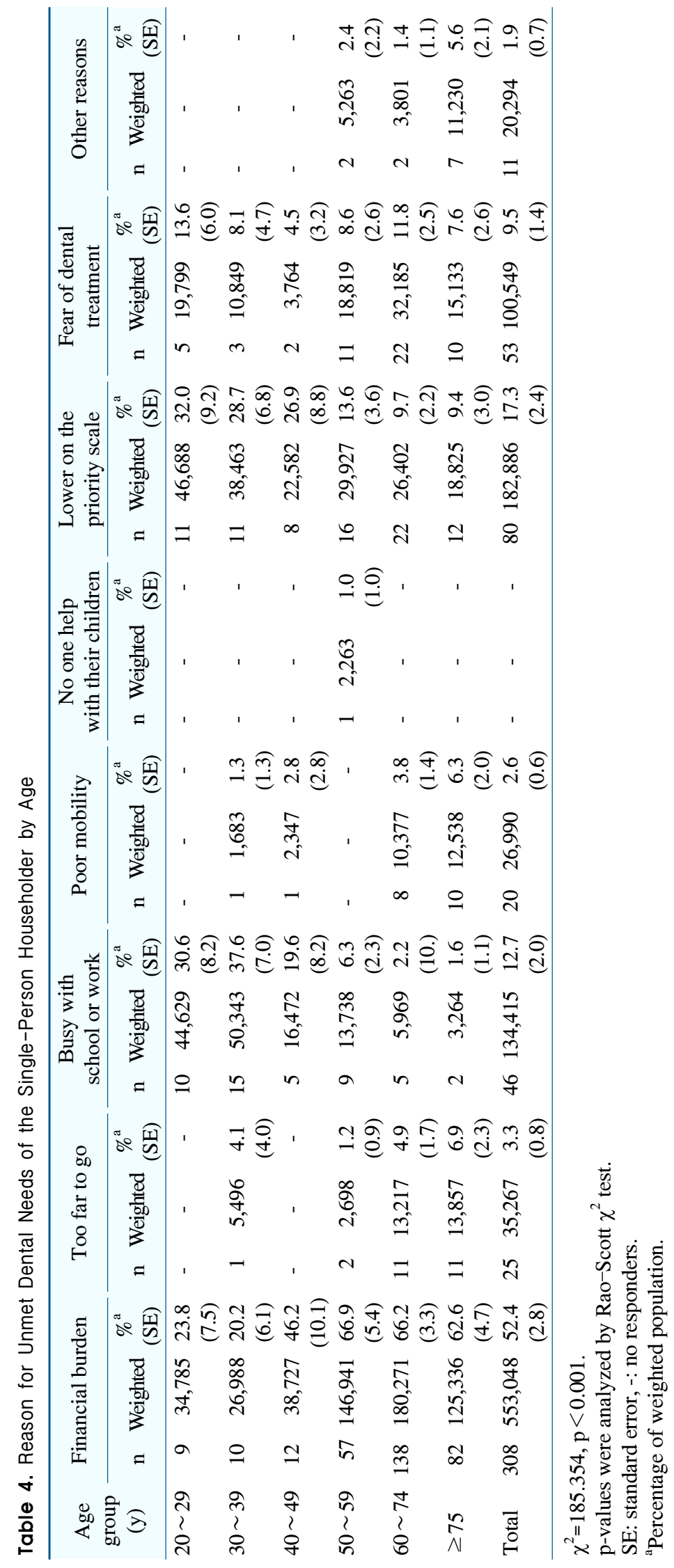


Table 5. Difference of Unmet Dental Needs by the Anderson Model

\begin{tabular}{|c|c|c|c|c|c|c|c|}
\hline \multirow{2}{*}{ Variable } & \multicolumn{3}{|c|}{ Yes } & \multicolumn{3}{|c|}{ No } & \multirow{2}{*}{$\chi^{2}(p)$} \\
\hline & $\mathrm{n}$ & Weighted & $\%^{\mathrm{a}}(\mathrm{SE})$ & $\mathrm{n}$ & Weighted & $\%^{\mathrm{a}}(\mathrm{SE})$ & \\
\hline Sex & & & & & & & $0.043(0.877)$ \\
\hline Male & 180 & 478,719 & $35.3(2.7)$ & 343 & 878,306 & $64.7(2.7)$ & \\
\hline Female & 364 & 576,996 & $34.8(1.7)$ & 693 & $1,082,002$ & $65.2(1.7)$ & \\
\hline \multicolumn{8}{|l|}{ Age group (y) } \\
\hline $20 \sim 29$ & 35 & 145,901 & $33.4(4.2)$ & 70 & 290,782 & $66.6(4.2)$ & $20.865(0.053)$ \\
\hline $30 \sim 39$ & 41 & 133,822 & $33.3(4.6)$ & 77 & 268,132 & $66.7(4.6)$ & \\
\hline $40 \sim 49$ & 28 & 83,892 & $27.4(4.6)$ & 82 & 222,112 & $72.6(4.6)$ & \\
\hline $50 \sim 59$ & 98 & 219,650 & $43.2(3.9)$ & 139 & 288,381 & $56.8(3.9)$ & \\
\hline $60 \sim 74$ & 124 & 176,182 & $39.8(3.1)$ & 214 & 266,650 & $60.2(3.1)$ & \\
\hline$\geq 75$ & 218 & 296,268 & $32.8(1.9)$ & 450 & 606,411 & $67.2(1.9)$ & \\
\hline Educational level & & & & & & & $10.824(0.138)$ \\
\hline$\leq$ Elementary school & 286 & 425,557 & $38.3(2.0)$ & 504 & 686,769 & $61.7(2.0)$ & \\
\hline Middle school & 51 & 81,200 & $29.1(4.0)$ & 111 & 197,704 & $70.9(4.0)$ & \\
\hline High school & 100 & 263,227 & $34.8(3.4)$ & 201 & 493,831 & $65.2(3.4)$ & \\
\hline$\geq$ University & 75 & 221,459 & $30.7(3.4)$ & 173 & 500,447 & $69.3(3.4)$ & \\
\hline No response & 32 & 64,271 & $44.1(6.6)$ & 47 & 81,557 & $55.9(6.6)$ & \\
\hline Hypertension & & & & & & & $0.170(0.920)$ \\
\hline Yes & 206 & 297,902 & $35.5(2.3)$ & 382 & 540,705 & $64.5(2.3)$ & \\
\hline No & 7 & 11,402 & $31.4(10.2)$ & 16 & 27,897 & $68.6(1.0)$ & \\
\hline No response & 331 & 746,410 & $34.9(1.9)$ & 638 & $1,394,706$ & $65.1(1.9)$ & \\
\hline Diabetes mellitus & & & & & & & $1.595(0.375)$ \\
\hline Yes & 80 & 130,749 & $38.3(3.6)$ & 149 & 210,716 & $61.7(3.6)$ & \\
\hline No & 2 & 1,710 & $17.7(12.1)$ & 6 & 7,956 & $82.3(12.1)$ & \\
\hline No response & 462 & 923,256 & $34.6(1.6)$ & 881 & $1,741,636$ & $65.4(1.6)$ & \\
\hline Smoking & & & & & & & $10.325(0.009)$ \\
\hline Yes & 134 & 347,607 & $41.2(3.0)$ & 193 & 496,316 & $58.8(3.0)$ & \\
\hline No & 410 & 708,108 & $32.6(1.5)$ & 843 & $1,463,992$ & $67.4(1.6)$ & \\
\hline Personal income level & & & & & & & $10.873(0.033)$ \\
\hline High & 45 & 99,242 & $25.4(4.0)$ & 127 & 291,876 & $74.6(4.0)$ & \\
\hline Middle & 257 & 494,810 & $35.0(2.1)$ & 504 & 917,429 & $65.0(2.1)$ & \\
\hline Low & 238 & 451,140 & $38(2.4)$ & 398 & 735,229 & $62.0(2.4)$ & \\
\hline No response & 4 & & & & & & \\
\hline Type of medical insurance & & & & & & & $10.705(0.056)$ \\
\hline Community based & 178 & 380,617 & $38.1(2.6)$ & 315 & 618,883 & $61.9(2.6)$ & \\
\hline Employed & 249 & 467,710 & $31.3(1.9)$ & 535 & $1,028,667$ & $68.7(1.9)$ & \\
\hline Medical aid & 89 & 150,211 & $38.3(4.1)$ & 145 & 241,514 & $61.7(4.1)$ & \\
\hline No response & 28 & 57,176 & $44.5(7.4)$ & 41 & 71,245 & $55.5(7.4)$ & \\
\hline Private health insurance & & & & & & & $4.321(0.304)$ \\
\hline Yes & 237 & 502,139 & $32.9(2.0)$ & 457 & $1,025,756$ & $67.1(12.0)$ & \\
\hline No & 298 & 533,369 & $37.6(2.1)$ & 563 & 885,634 & $62.4(2.1)$ & \\
\hline No response & 9 & 20,207 & $29.2(11.4)$ & 16 & 48,918 & $70.8(11.4)$ & \\
\hline Residence & & & & & & & $0.000(0.995)$ \\
\hline Dong & 419 & 852,794 & $35(1.7)$ & 802 & $1,583,233$ & $65(1.7)$ & \\
\hline Eup, myeon & 125 & 202,921 & $35(3.1)$ & 234 & 377,075 & $65(3.1)$ & \\
\hline Regular oral check-ups & & & & & & & $18.802(0.001)$ \\
\hline Yes & 84 & 182,971 & $26.1(2.8)$ & 245 & 518,346 & $73.9(2.8)$ & \\
\hline No & 459 & 870,416 & $37.6(1.7)$ & 791 & $1,441,963$ & $62.4(1.7)$ & \\
\hline No response & 1 & 2,329 & $100(0.0)$ & & & & \\
\hline
\end{tabular}


Table 5. Continued

\begin{tabular}{|c|c|c|c|c|c|c|c|}
\hline \multirow{2}{*}{ Variable } & \multicolumn{3}{|c|}{ Yes } & \multicolumn{3}{|c|}{ No } & \multirow{2}{*}{$\chi^{2}(\mathrm{p})$} \\
\hline & $\mathrm{n}$ & Weighted & $\%^{\mathrm{a}}(\mathrm{SE})$ & $\mathrm{n}$ & Weighted & $\%^{\mathrm{a}}(\mathrm{SE})$ & \\
\hline Toothbrushing per day & & & & & & & $0.073(0.799)$ \\
\hline Yes & 528 & $1,026,850$ & $35.0(1.5)$ & 1,007 & $1,908,451$ & $65.0(1.5)$ & \\
\hline No & 16 & 288,645 & $37.0(7.9)$ & 28 & 49,097 & $63.0(7.9)$ & \\
\hline Economic activity & & & & & & & $3.532(0.298)$ \\
\hline Yes & 239 & 518,214 & $33.9(2.1)$ & 436 & $1,011,234$ & $66.1(2.1)$ & \\
\hline No & 274 & 474,157 & $35.3(2.1)$ & 555 & 870,718 & $64.7(2.1)$ & \\
\hline No response & 31 & 63,344 & $44.7(6.8)$ & 45 & 78,356 & $55.3(6.8)$ & \\
\hline Perceived oral health & & & & & & & $129.878(<0.001)$ \\
\hline Good & 36 & 69,273 & $17.7(3.2)$ & 181 & 321,016 & $82.3(3.2)$ & \\
\hline Normal & 115 & 239,942 & $22.2(2.2)$ & 404 & 838,748 & $77.8(2.2)$ & \\
\hline Poor & 338 & 631,099 & $48.5(2.5)$ & 384 & 669,240 & $51.5(2.5)$ & \\
\hline No response & 55 & 115,401 & $46.8(4.6)$ & 67 & 131,305 & $53.2(4.6)$ & \\
\hline Toothache within a year & & & & & & & $47.806(<0.001)$ \\
\hline Yes & 252 & 488,327 & $43.4(2.6)$ & 322 & 635,702 & $56.6(2.6)$ & \\
\hline No & 237 & 451,987 & $27.5(1.8)$ & 647 & 119,330 & $72.5(1.8)$ & \\
\hline No response & 55 & 115,401 & $46.8(4.6)$ & 67 & 131,305 & $53.2(4.6)$ & \\
\hline Speaking difficulty & & & & & & & $54.150(<0.001)$ \\
\hline Comfortable & 288 & 636,100 & $29.9(1.8)$ & 730 & $1,488,288$ & $70.1(1.8)$ & \\
\hline Unawareness & 83 & 145,890 & $38.6(3.8)$ & 147 & 231,681 & $61.4(3.8)$ & \\
\hline Uncomfortable & 173 & 273,725 & $53.5(3.1)$ & 158 & 237,447 & $46.5(3.1)$ & \\
\hline Chewing difficulty & & & & & & & $138.998(<0.001)$ \\
\hline Yes & 320 & 530,954 & $55.9(2.3)$ & 287 & 419,596 & $44.1(2.3)$ & \\
\hline No & 224 & 524,760 & $25.4(1.7)$ & 749 & $1,540,722$ & $74.6(1.7)$ & \\
\hline Periodontal disease & & & & & & & $11.744(0.010)$ \\
\hline Yes & 215 & 386,822 & $39.6(2.4)$ & 330 & 588,815 & $60.4(2.4)$ & \\
\hline No & 238 & 499,666 & $31.2(2.0)$ & 546 & $1,104,202$ & $68.8(2.0)$ & \\
\hline No response & 91 & 169,227 & $38.8(3.4)$ & 160 & 267,290 & $61.2(3.4)$ & \\
\hline Untreated dental caries & & & & & & & $64.843(<0.001)$ \\
\hline Yes & 215 & 437,038 & $47.3(2.8)$ & 236 & 486,097 & $52.7(2.8)$ & \\
\hline No & 275 & 505,555 & $27.3(1.8)$ & 734 & $1,344,111$ & $72.7(1.8)$ & \\
\hline No response & 54 & 113,122 & $46.5(4.6)$ & 66 & 130,099 & $53.5(4.6)$ & \\
\hline Limitation of activitiy & & & & & & & $31.715(<0.001)$ \\
\hline Yes & 7 & 13,162 & $86.0(13.1)$ & 1 & 2,142 & $14.0(13.1)$ & \\
\hline No & 114 & 206,097 & $48.9(4.0)$ & 134 & 215,773 & $51.1(4.1)$ & \\
\hline No response & 423 & 836,456 & $32.4(1.5)$ & 901 & $1,742,393$ & $67.6(1.5)$ & \\
\hline
\end{tabular}

p-values were analyzed by Rao-Scott $\chi^{2}$ test.

SE: standard error.

${ }^{\mathrm{a}}$ Percentage of weighted population.

experienced unmet dental needs was $41.2 \%$ and $32.6 \%$, respectively, showing that smokers had greater unmet dental needs than non-smokers $(p=0.009)$.

Among the enabling factors, income level and regular oral health screening showed differences in unmet dental needs experiences. The lower-income level group showed the highest rate of unmet dental needs experiences at $38 \%$ $(\mathrm{p}=0.033)$, while the group that did not receive regular oral health screening showed a higher rate of unmet dental needs experiences at $37.6 \%(\mathrm{p}=0.001)$.

Among the need factors, all factors, except periodontal disease, showed significant differences. The group with poor subjective oral health status showed the highest rate of unmet dental needs experiences at $48.5 \%(\mathrm{p}<0.001)$; the group with experience of toothache in the past year showed a higher rate of unmet dental needs experiences at 
43.4\% ( $\mathrm{p}<0.001)$; the group with speech impairment showed a higher rate of unmet dental needs experiences at $53.5 \%(\mathrm{p}<0.001)$; the group with chewing discomfort showed a higher rate of unmet dental needs experiences at $55.9 \%(\mathrm{p}<0.001)$; the group with permanent teeth dental caries experience showed a higher rate of unmet dental needs experiences at $47.3 \%(\mathrm{p}<0.001)$; and in the group with activity limitations, those with limitations due to dental and oral issues showed the highest rate of unmet dental needs experiences at $86.0 \%(\mathrm{p}<0.001)$.
5. Influencing factors of unmet dental needs experiences among single-person households

The influencing factors of unmet dental needs experiences among single-person households were identified as smoking status, income level, chewing discomfort, and activity limitations (Table 6).

Smokers, the lower income level group, the group with chewing discomfort, and the group with activity limitations showed a higher rate of unmet dental needs experiences. Smokers showed a 2.75-fold higher rate of unmet dental needs experiences than non-smokers

Table 6. Factors Influencing the Unmet Dental Needs

\begin{tabular}{|c|c|c|c|c|c|}
\hline Variable & $\mathrm{B}$ & SE & Adjusted OR & $95 \% \mathrm{CI}$ & p-value \\
\hline \multicolumn{6}{|l|}{ Smoking } \\
\hline Yes & -1.011 & 0.371 & 2.746 & $0.176 \sim 0.754$ & 0.007 \\
\hline No (ref.) & & & 1.000 & & \\
\hline \multicolumn{6}{|l|}{ Personal income level } \\
\hline Low & -1.324 & 0.806 & 3.759 & $0.055 \sim 1.297$ & 0.101 \\
\hline Middle & -1.665 & 0.819 & 5.287 & $0.038 \sim 0.946$ & 0.043 \\
\hline High (ref.) & & & 1.000 & & \\
\hline \multicolumn{6}{|l|}{ Regular oral check-ups } \\
\hline No & 0.379 & 0.445 & 1.505 & $0.608 \sim 3.504$ & 0.396 \\
\hline Yes (ref.) & & & 1.000 & & \\
\hline \multicolumn{6}{|l|}{ Perceived oral health } \\
\hline Poor & 1.051 & 0.759 & 2.860 & $0.644 \sim 12.710$ & 0.167 \\
\hline Normal & 0.064 & 0.775 & 1.066 & $0.232 \sim 4.897$ & 0.934 \\
\hline Good (ref.) & & & 1.000 & & \\
\hline \multicolumn{6}{|l|}{ Toothache within a year } \\
\hline Yes & -0.351 & 0.378 & 1.420 & $0.335 \sim 1.480$ & 0.354 \\
\hline No (ref.) & & & 1.000 & & \\
\hline \multicolumn{6}{|l|}{ Speaking difficulty } \\
\hline Comfortable & -0.513 & 0.439 & 1.669 & $0.253 \sim 1.419$ & 0.243 \\
\hline Unawareness & 0.563 & 0.483 & 0.569 & $0.680 \sim 4.539$ & 0.244 \\
\hline Uncomfortable (ref.) & & & 1.000 & & \\
\hline \multicolumn{6}{|l|}{ Chewing difficulty } \\
\hline Yes & -1.183 & 0.441 & 3.269 & $0.129 \sim 0.729$ & 0.008 \\
\hline No (ref.) & & & 1.000 & & \\
\hline \multicolumn{6}{|l|}{ Periodontal disease } \\
\hline Yes & -0.136 & 0.366 & 1.146 & $0.425 \sim 1.791$ & 0.709 \\
\hline No (ref.) & & & 1.000 & & \\
\hline \multicolumn{6}{|l|}{ Untreated dental caries } \\
\hline Yes & -0.155 & 0.437 & 1.166 & $0.363 \sim 2.023$ & 0.723 \\
\hline No (ref.) & & & 1.000 & & \\
\hline \multicolumn{6}{|l|}{ Limitation of activity } \\
\hline Yes & -2.060 & 1.018 & 7.866 & $0.017 \sim 0.943$ & 0.044 \\
\hline No (ref.) & & & 1.000 & & \\
\hline
\end{tabular}

By complex samples regression adjusted for decayed, missing, filled tooth (DMFT) index.

SE: standard error, OR: odds ratio, CI: confidence interval, ref.: reference. 
$(p=0.007)$. The lower-income level group showed a 5.29-fold higher rate of unmet dental needs experiences than the middle-income level group $(p=0.043)$, but no statistically significant differences compared to the upper-income level group. The group with chewing discomfort showed a 3.27-fold higher rate of unmet dental needs experiences than the group with no discomfort $(\mathrm{p}=0.008)$, while the group with activity limitations showed a 7.87-fold higher rate of unmet dental needs experiences than the group with no limitations $(p=0.044)$.

\section{Discussion}

The rate of unmet dental needs experiences among single-person households decreased in the order of 2014, 2013, and 2015, showing an inconsistent pattern. Among the predisposing, enabling, and need factors, differences in unmet dental needs experiences among single-person households were found based on smoking status, income level, regular oral health screening, subjective oral health status, toothache in the past year, speech impairment, chewing discomfort, permanent teeth dental caries experience, and activity limitations. Smokers, the lowerincome level group, the group with chewing discomfort, and the group with activity limitations showed a higher rate of unmet dental needs experiences, with these influencing factors appearing in the following order of importance: activity limitations, income level, chewing discomfort, and smoking status.

The groups with chewing discomfort and activity limitations showed a higher rate of unmet dental needs experiences, which is consistent with the findings in previous studies ${ }^{1,9)}$. In particular, the group with chewing discomfort is meaning from the standpoint that it is an indicator that can be used to objectively measure the unmet dental needs experiences of the subjects. In Korea, dental services are usually performed in dental hospitals, and thus the group with activity limitations would inevitably have a high rate of unmet dental needs experiences. Given that $62.9 \%$ of the study subjects were elderly people aged 60 or older and that $90 \%$ of the subjects who gave "being frail/health issues" as the subjective reason for unmet dental needs were also elderly people aged 60 or older, measures regarding accessibility to dental hospitals should focus especially on elderly people in single-person households.

With respect to income level, the lower-income group showed the highest rate of unmet dental needs experiences, with such experiences decreasing with increases in income level; this was consistent with the findings in previous studies $^{21-23)}$. Considering that $54 \%$ of the respondents stated that they did not receive the necessary dental care services due to economic reasons, the cost of dental care services seems to act as an impediment to meeting the needs for dental services. The goal of any public health care system is to guarantee the health and quality of life of its citizens by providing health care services based on the need for such services rather than the ability to pay ${ }^{24)}$. Despite the fact that providing the right dental services at the right time is important, dental services can result in an $83.5 \%$ out-of-pocket cost due to many high-cost items that are not covered by insurance ${ }^{24)}$, which can cause a problem of impeding accessibility for those on a lowerincome level. In particular, because people perceive oral diseases to be less life-threatening that other medical diseases, they tend to underestimate the urgency of oral diseases ${ }^{1)}$. From preventive dental care, such as scaling, sealant treatment, and resin filling, to rehabilitative dental care, such as dentures and implants, the range of dental services covered by insurance is expected to continue to expand. In the future, if dental services are compared by the year of implementation, the effect may be greater accessibility.

The fact that smokers have a higher rate of unmet dental needs experiences than non-smokers is consistent with the results for members of all types of households. Among members of all types of households, smokers showed a 1.186-fold higher rate of unmet dental needs experiences than non-smokers ${ }^{21)}$, while among single-person households, smokers showed a 2.75-fold higher rate of unmet dental needs experiences than non-smokers, indicating that smokers from a single-person household are more likely to experience unmet dental needs than smokers from other types of households. Smokers are known to utilize outpatient services less frequently owing to psychological factors, such as subjective health status and stress ${ }^{25}$. 
Because smoking itself is a key factor that affects oral health ${ }^{26}$, follow-up studies are needed with consideration for the mediating variables and psychological characteristics associated with the process by which smoking status affects unmet dental needs experiences.

In addition, regular oral health screening, subjective oral health status, toothache in the past year, speech impairment, and permanent dental care experience showed statistically significant differences in unmet dental needs as compared to their counterparts, but the logistic regression analysis did not show a statistically significant influence. Such findings contradicted the results from analyzing the factors associated with unmet dental needs experiences among all types of households ${ }^{19}$, which may be attributable to the internal heterogeneity and the characteristics of single-person households. The demographic characteristics of single-person households have greater internal heterogeneity than those of other types of households ${ }^{10)}$. The increased number of singleperson households may be attributable to changes in social patterns, such as changes in household structure, late marriage, and an increase in the population of those who are single, as well as changes in the population structure, such as a low birth rate and population aging. Moreover, various differences in sex characteristics, marital status, and area of residence are found among different age groups ${ }^{10,11)}$. Because the present study conducted the analyses without adjusting for factors that may cause internal heterogeneity, such as sex, age, area of residence, and income level of single-person household members, a follow-up study that considers these factors is recommended.

Various measures are needed to reduce the unmet dental needs experiences among single-person households. Specific strategies that include utilization of a part-time dentist system for long-term care facilities, home-visit oral hygiene services, and nearby dentist referral services should be devised to refer and provide dental services to single-person household members with activity limitations. In particular, the fact that most of the single-person household members with activity limitations are elderly people aged 60 or over should be taken into account. When a professional oral hygiene program was implemented for elderly residents in long-term care facilities, the program had the effect of reducing the dental plaque index, halitosis, and tongue coatings, while increasing saliva flow $^{27)}$. Moreover, health insurance coverage should be expanded to ensure equity in dental services for singleperson household with a low-income level, while the reason for these same households having a high smoking rate should be analyzed from multiple perspectives; mediating variables associated with the process by which smoking status affects unmet dental needs experiences should also be analyzed to actively recommend policies that can ultimately reduce the smoking rate.

Because the present study analyzed data from the 6th KNHANES that used a self-reporting format, the dental services that medical professionals determine are needed could not be measured objectively. Moreover, because the questionnaire did not include questions on the experience of dental needs that the subjects wanted to meet but which could not be met, this aspect could not be measured. As a limitation inherent in regression analysis, influencing factors of unmet dental needs were analyzed crosssectionally and individually. Accordingly, a follow-up study is needed to analyze the complex relationships between various factors with consideration of factors that cause internal heterogeneity, such as sex, age, area of residence, and income level. Despite these limitations, the significance of the present study can be found in the fact that it attempted to allow the results to be interpreted as results for all single-person households, such as accounting for weighted values; it also analyzed the influencing factors of the utilization of dental services according to the Anderson model, a typical socio-behavioral model used as an analytic framework for behavioral prediction and identification of the current status of unmet dental needs among single-person households to provide basic data on unmet dental needs among single-person households.

\section{Notes}

\section{Conflict of interest}

No potential conflict of interest relevant to this article was reported. 


\section{Ethical approval}

The present study was conducted with a review exemption from the Institutional Review Board at Namseoul University (No. 1041479-HR-201809-010).

\section{ORCID}

Dong-Hwi Kim, https://orcid.org/0000-0002-3181-0917

Hyeongmi Kim, https://orcid.org/0000-0001-5502-7460

\section{References}

1. Ahn ES, Shin MS: Factors related to the unmet dental care needs of adults with dental pain. J Dent Hyg Sci 16: 355-360, 2016. https://doi.org/10.17135/jdhs.2016.16.5.355

2. Donabedian A: Aspects of medical care administration: specifying requirements for health care. Harvard University Press, Boston, 1973.

3. Alonso J, Orfila F, Ruigómez A, Ferrer M, Antó JM: Unmet health care needs and mortality among Spanish elderly. Am J Public Health 87: 365-370, 1997.

4. Huh SI, Kim SJ: Unmet needs for health care among Korean adults: differences across age groups. Korean J Health Econ Policy 13: 1-16, 2007.

5. Diamant AL, Hays RD, Morales LS, et al.: Delays and unmet need for health care among adult primary care patients in a restructured urban public health system. Am J Public Health 94: 783-789, 2004.

6. KOrean Statistical Information Service (KOSIS): Status of medical institutions by type establishment. Retrieved December 28, 2018, from http://kosis.kr/statHtml/statHtml. do? orgId=354\&tblId=DT_HIRA43\&vw_cd=MT_ZTITLE $\&$ list_id=354_MT_DTITLE\&seqNo=\&lang_mode $=$ ko\&langu age=kor\&obj_var_id=\&itm_id=\&conn_path=MT_ZTITLE ( 2019, February 11).

7. Shin HS, Hong SY: The supply and demand for dentists in Korea. Health Soc Welf Rev 27: 81-102, 2007.

8. Retrieved November 12, 2018, from http://www.cdc.go.kr/ $\mathrm{CDC} / \mathrm{mobile} / \mathrm{CdcKrContentView}$.jsp?cid=60940\&menuIds= HOME001-MNU1130-MNU1639-MNU1748-MNU1752.ex $\operatorname{cel}(2017)$.

9. Ministry of Health and Welfare: Social security by statistics. Ministry of Health and Welfare, Sejong, p.14, 2017.

10. Son HK: Solo economy analysis. Statistics Korea, Daejeon, pp.153-173, 2017.

11. Choi JP, Lee YJ, Ryu JW, Byun NH, Moon GJ: School conversion strategy for affordable housing. J Archit Inst Korea Plan Des 26: 3-11, 2010.

12. Shim SB, Lee KM, Lee KW, Jeong JM, Park YS, Shin SY: Cardiovascular risk of one-person households. Korean J Fam Pract 8: 8-14, 2018. https://doi.org/10.21215/kjfp.2018.8.1.8

13. Ha JK, Lee SL: The effect of health-related habitual consumption and lifetime on subjective health of one person households: focusing on comparison between non-one person households and generations. Fam Environ Res 55: 141-152, 2017. https://doi.org/10.6115/fer.2017.011

14. Jeong KM, Nam SH, Jeong EG, Lee GH, Lee YG, Kim JS: Policy implications of changes in family structure: focused on the increase of single person households in Korea. Korea Institute for Health and Social Affairs (KIHASA), Seoul, pp.138-139, 2012.

15. National Health Insurance, Korea Institute for Health and Social Affairs: Korea health system based on healthcare indicators-2016 Korea medical panel in-depth analysis report. National Health Insurance Health Insurance Policy Institute, Wonju, 2016.

16. Lee HJ, Huh SI: Unmet health care needs and impact of type of household among the elderly in Korea. Korean J Health Econ Policy 23: 85-108, 2017.

17. Moon JH, Kang MN: The prevalence and predictors of unmet medical needs among the elderly living alone in Korea: an application of the behavioral model for vulnerable populations. Health Soc Welf Rev 36: 480-510, 2016. https://doi.org/10.15709/hswr.2016.36.2.480

18. Jeong GC, Lee HS, Kim SN: A study on the dental services utilization by application of the Andersen model in students of some Korean university. J Korean Acad Oral Health 27: 357-373, 2003.

19. Kim DH, Seo YJ: Factors influencing the regular oral check-ups: based on the data of the 2014 Korea National Health and Nutrition Examination Survey. J Dent Hyg Sci 17: 323-332, 2017. https://doi.org/10.17135/jdhs.2017.17.4.323

20. Lee DY, Park JD: A study on the factors for the elderly living alone at home to determine their participation in a health promotion activity program: with the application of Anderson model. Soc Welf Policy 38: 1-23, 2011.

21. Yoo SH, Park JS, Kim YM: A decision-tree analysis of 
influential factors and reasons for unmet dental care in Korean adults. Health Soc Welf Rev 37: 294-335, 2017. https://doi.org/10.15709/hswr.2017.37.4.294

22. Kim SJ, Huh SI: Financial burden of health care expenditures and unmet needs by socioeconomic status. Korean J Health Econ Policy 17: 47-70, 2011.

23. Moon SE, Song AH: Factors affecting unmet dental care needs of Korean: the 6th Korean national health and nutritional examination survey. J Korean Soc Dent Hyg 16: 769-774, 2016.

https://doi.org/10.13065/jksdh.2016.16.05.767

24. Organisation for Economic Co-operation and Development
(OECD): Health at a glance 2013: OECD indicators. OECD Publishing, Paris, pp.146-147, 2013.

25. Shin MS, Lee WJ: Health behavior associated with outpatient utilization. J Korea Content Assoc 13: 342-353, 2013. https://doi.org/10.5392/JKCA.2013.13.05.342

26. Ju OJ, Kim IJ: Comparison of dental health capacity between smokers and nonsmokers. J Dent Hyg Sci 16: 200-209, 2016. https://doi.org/10.17135/jdhs.2016.16.3.200

27. Lee KY, Lim SR: Effect of professional oral healthcare program on the oral status of elderly residents in long-term care facilities. J Dent Hyg Sci 16: 432-441, 2016. https://doi.org/10.17135/jdhs.2016.16.6.432 